\title{
Análise de adulteração da Cúrcuma longa L. em pó comercializada em Campina Grande - PB e Pocinhos -PB
}

\author{
Adulteration analysis of Curcuma longa L. powder sold in Campina Grande - PB and Pocinhos-PB \\ Análisis de adulteración del polvo de Curcuma longa L. vendido en Campina Grande - PB y
}

Pocinhos-PB

Recebido: 18/05/2021 | Revisado: 27/05/2021 | Aceito: 31/05/2021 | Publicado: 15/06/2021

\author{
Mayanny Celly de Sales Mélo \\ ORCID: https://orcid.org/0000-0002-1200-0353 \\ Centro Universitário Unifacisa, Brasil \\ E-mail: mayannycelly@gmail.com \\ Patricia de Luna Rodrigues \\ ORCID: https://orcid.org/0000-0001-6994-8367 \\ Centro Universitário Unifacisa, Brasil \\ E-mail: patriciarodrigues23.luna@gmail.com \\ Vanessa Cristina de Melo Silva \\ ORCID: https://orcid.org/0000-0003-2111-881X \\ Centro Universitário Unifacisa, Brasil \\ E-mail: vanessacmelosilva@gmail.com \\ Marina Suênia de Araújo Vilar \\ ORCID: https://orcid.org/0000-0003-4957-0752 \\ Centro Universitário UNIFACISA, Brasil \\ E-mail: peritaquimica@yahoo.com.br \\ Daniela de Araújo Vilar \\ ORCID: https://orcid.org/0000-0002-0264-0381 \\ Centro Universitário Unifacisa, Brasil \\ E-mail: dani_1011@yahoo.com.br
}

\begin{abstract}
Resumo
A Cúrcuma longa pertence à família Zingiberaceae, popularmente conhecida como açafrão, açafrão da terra, gengibre amarelo e raiz de sol. Consumida principalmente na forma de pó seco, utilizada com diversas finalidades, principalmente na medicina tradicional e moderna. Como consequência do seu elevado valor comercial, a cúrcuma tem sido frequentemente adulterada com os mais diversos materiais e estratégias. Neste sentido, o objetivo desse trabalho foi analisar possíveis adulterações em amostras de cúrcuma em pó comercializadas nas cidades de Campina Grande e Pocinhos na Paraíba. Para isso, foram realizados os testes de ativação do amido e reação do lugol em 10 amostras adquiridas em diferentes pontos comerciais das cidades citadas. No teste de ativação do amido apenas uma amostra resultou em uma solução encorpada indicando a presença de amido. Já no teste de reação do lugol 4 amostras apresentaram uma coloração azul escuro/violeta que indica a presença de amido. Observou-se que a adulteração foi encontrada em maior percentual nas amostras comercializadas em feiras livres. Diante dos resultados observa-se que a detecção de adulterantes torna-se assim, uma questão de elevada importância para garantir a proteção do consumidor contra práticas fraudulentas. Desta forma, conclui-se que os resultados deste trabalho revelaram fraude em algumas amostras e evidencia a importância da adoção de medidas de controle e fiscalização para a prevenção de prejuízos econômicos e riscos à saúde do consumidor.
\end{abstract}

Palavras-chave: Fitoterapia; Plantas medicinais; Adulteração; Curcuma longa L.

\begin{abstract}
Cúrcuma longa belongs to the Zingiberaceae family, popularly known as turmeric, turmeric, yellow ginger and sun root. Consumed mainly in the form of dry powder, used for several purposes, mainly in traditional and modern medicine. As a consequence of its high commercial value, turmeric has often been adulterated with the most diverse materials and strategies. In this sense, the objective of this work was to analyze possible adulterations in samples of turmeric powder sold in the cities of Campina Grande and Pocinhos in Paraíba. For this, the starch activation and lugol reaction tests were carried out on 10 samples acquired at different commercial points in the cities mentioned. In the starch activation test, only one sample resulted in a full-bodied solution indicating the presence of starch. In the lugol reaction test, 4 samples showed a dark blue / violet color that indicates the presence of starch. It was observed that adulteration was found in a higher percentage in samples sold in open markets. In view of the results, it is observed that the detection of adulterants thus becomes an issue of high importance to ensure consumer protection
\end{abstract}


against fraudulent practices. Thus, it is concluded that the results of this work revealed fraud in some samples and highlights the importance of adopting control and inspection measures to prevent economic losses and risks to consumer health.

Keywords: Phytotherapy; Medicinal plants; Adulteration; Long turmeric L.

\section{Resumen}

Cúrcuma longa pertenece a la familia Zingiberaceae, popularmente conocida como cúrcuma, cúrcuma, jengibre amarillo y raíz de sol. Consumido principalmente en forma de polvo seco, utilizado para diversos fines, principalmente en la medicina tradicional y moderna. Como consecuencia de su alto valor comercial, la cúrcuma a menudo ha sido adulterada con los más diversos materiales y estrategias. En este sentido, el objetivo de este trabajo fue analizar posibles adulteraciones en muestras de cúrcuma en polvo comercializadas en las ciudades de Campina Grande y Pocinhos en Paraíba. Para ello, se realizaron las pruebas de activación de almidón y reacción de lugol en 10 muestras adquiridas en diferentes puntos comerciales de las ciudades mencionadas. En la prueba de activación del almidón, solo una muestra dio como resultado una solución con cuerpo que indica la presencia de almidón. En la prueba de reacción de lugol, 4 muestras mostraron un color azul oscuro / violeta que indica la presencia de almidón. Se observó que la adulteración se encontró en un mayor porcentaje en las muestras vendidas en mercados abiertos. A la vista de los resultados, se observa que la detección de adulterantes se convierte así en un tema de gran importancia para asegurar la protección del consumidor frente a prácticas fraudulentas. Así, se concluye que los resultados de este trabajo revelaron fraude en algunas muestras y resalta la importancia de adoptar medidas de control y fiscalización para la prevención de pérdidas económicas y riesgos para la salud de los consumidores.

Palavras clave: Fitoterapia; Plantas medicinales; Adulteración; Curcuma longa L.

\section{Introdução}

Desde os tempos remotos, as plantas possuem um papel significativo no desenvolvimento da sociedade, fazendo parte da vida humana desde os primórdios. Logo, o uso de plantas medicinais no tratamento e na cura de patologias se distende até o cenário atual. Visto que, ao longo do tempo as plantas foram uma das principais fontes de tratamento na terapêutica dos seres humanos (Calixto, 2019). Dessa maneira, o uso de plantas medicinais para fins terapêuticos é uma prática convencional empregada pela população brasileira, sendo muitas das vezes o único recurso disponível na atenção básica de saúde (Pedrete, 2018).

Através dos incentivos do Ministério da Saúde e do conhecimento empírico, a utilização das plantas no cuidado à saúde vem se ampliando consideravelmente. Diante de tal importância, surgi à elaboração e publicação da Relação de Plantas Medicinais de Interesse do SUS (RENISUS), no qual, foram listadas 71 espécies vegetais, dentre elas, estão incluídas as plantas utilizadas pela população confirmadas cientificamente (Brasil, 2009). Em vista disso, a criação dessa lista favoreceu o desenvolvimento de medicamentos fitoterápicos, tornando-se um passo importantíssimo para o desenvolvimento de mais pesquisas sobre as plantas medicinais (Muniz, et al. 2013; Lucas, et al. 2016).

Incluída na RENISUS, a Cúrcuma longa L. pertencente à família Zingiberaceae, conhecida por suas inúmeras aplicações sejam elas terapêuticas, com diversas atividades farmacológicas; utilizada na culinária como tempero, conservante, corante alimentar; em cosméticos ou em práticas religiosas. Sua utilização é caracterizada por diversas finalidades e benefícios à saúde, agindo em vários tipos de patologias como método de profilaxia, especialmente seu emprego contra artrite, diabetes além de hepatite e malária. Dentre as atividades farmacológicas atribuídas a cúrcuma ressalta-se suas ações antimicrobiana, anticancerígena, anticoagulante, antifúngica, antitumoral, antimalárica, imunomoduladora, cicatrizante e neuroprotetora (Moretes \& Geron, 2019).

Segundo Alonso (2016), inicialmente a Cúrcuma longa era apreciada por seu valor alimentício, propriedades similares ao gengibre, logo depois, foi reconhecida por suas diversas propriedades etnomedicinais que incluem: atividade hepática, gastroprotetora, anti-HIV, hipolipemiante, hipoglicemiante, antiagregante, dermatológica, oftalmológica, em oncologia, como em diversos sistemas: sendo eles o sistema respiratório, sistema reprodutor, sistema digestório e no sistema nervoso central. 
Entretanto, diante da sua vasta aplicabilidade e seu grande potencial farmacológico, principalmente pelo seu efeito anti-inflamatório, o que de fato tem preocupado é que os produtos naturais vendidos a granel podem causar danos à saúde da população quando não se sabe a sua composição, se fazendo necessário à realização de estudos que tragam tais informações aos consumidores (Silva Neto; Leite, Aguiar, 2020).

Com isso, a fraude pode ser definida como toda e qualquer intervenção em alimentos elaborada em condições contrárias às especificações legais. Provocam prejuízos de ordem biológica e econômica ao consumidor, além de serem antiéticos e conflitarem com a legislação (Spink; Moyer, 2011).

Essas fraudes em alimentos são passíveis de ocorrer em todas as áreas, seja na comercialização entre empresas ou na comercialização para o consumidor final. O desafio dos órgãos fiscalizadores é desenvolver técnicas de identificação tão ou mais eficazes quanto às utilizadas pelos falsificadores.

Em virtude disso, a fraude de alimento pode advir de fatores como a adulteração, troca parcial de um item e a utilização de falsas declarações referente à composição do produto, origem ou de qualidade do alimento (Barreto, et al. 2018).

Em geral, a causa principal da fraude é econômica, já que os adulterantes utilizados possuem baixo custo ou porque são empregadas substâncias químicas para camuflar a qualidade dos produtos (Galvin-king; Haughey; Elliott, 2018). Com isso, estima-se que a fraude alimentar possua um custo anual global de aproximadamente 49 bilhões de dólares, onde cerca de 10\% dos produtos alimentares que compramos tenham algum tipo adulteração (GMA-SEF, 2018).

Quando relacionado com as especiarias, a sua produção acontece na maioria das vezes em locais que as práticas higiênico-sanitárias e de controle no processamento industrial, ainda são bem restritas e precárias favorecendo a contaminação acidental com materiais estranhos, ou até mesmo intencionais com a utilização de materiais com aspecto similar, mas de valor econômico bastante inferior, sendo ela, a mais praticada, com o intuito unicamente de ampliar os lucros (Sousa, 2017).

Sabe-se que alguns produtos manufaturados que são vendidos em menor escala e de forma caseira, em feiras públicas e supermercados, muitas vezes, apresentam apenas uma embalagem de acondicionamento, não contendo quaisquer informações nutricionais ao consumidor (Almeida, et al. 2017). Com a ocultação ou até mesmo sem a menção dessas informações a respeito da composição dos alimentos os consumidores não conseguem saber de fato o que estão ingerindo (Silva Neto; Leite, Aguiar, 2020).

Assim, contribuindo para que diversas especiarias, dentre elas a cúrcuma, sejam submetidas às adulterações e fraudes, estando entre os 25 alimentos com maior número de registros de fraudes (Pauli-Yamad, et al.2020). Podendo ocorrer pela adição ou troca dos componentes. Sendo sua detecção um desafio constante, nos quais, grandes prejuízos estão relacionados em decorrência dessas práticas (Moore; Spink; Lipp, 2012; Johnson, 2014; Cérou, 2018).

Geralmente o objetivo dessas adulterações na composição do produto é de gerar ganhos econômicos ao fabricante, constituindo fraude. Em alguns casos, essas adulterações podem acarretar inúmeras consequências para o consumidor, podendo ser elas, doenças ou óbito (GFSI, 2018). Logo, a RDC ANVISA nº 259, de 20 de setembro de 2002 aprova o regulamento técnico sobre rotulagem de alimentos embalados, sendo empregada a lista de ingredientes dos produtos como valor de referência (Brasil, 2002).

Dessa forma, o desenvolvimento deste estudo torna-se relevante diante do interesse público na qualidade dos produtos naturais consumidos, o qual vem aumentado significativamente nas últimas décadas. Onde tem se buscado aperfeiçoar as regulamentações e as exigências sobre a produção e comercialização de produtos medicinais, a fim de, garantir a segurança e a eficácia dos mesmos, possibilitando sua utilização sem maiores riscos à saúde dos seus usuários e justificando, assim, a importância na realização desse estudo.

Diante do exposto, o trabalho possui o objetivo de analisar uma possível adulteração em amostras de Cúrcuma longa L. comercializadas em Campina Grande-PB e Pocinhos-PB. 


\section{Metodologia}

Trata-se de uma pesquisa do tipo experimental com variáveis quantitativas e qualitativas, onde por sua vez o analista é um agente ativo, entretanto não comportando apenas como observador. Os dados da pesquisa são obtidos por meio de análises (Gil, 2007). As amostras de cúrcuma em pó foram adquiridas nas cidades de Campina Grande ( $7^{\circ} 13^{\prime} 50^{\prime \prime} \mathrm{S}, 35^{\circ} 52^{\prime}$ '52" W, $\mathrm{PB}$, Brasil) e Pocinhos (7 04' 37" S 36 03' 39" O, PB, Brasil) através de feiras livres, lojas de produtos naturais e supermercados. Os procedimentos e análises foram realizadas no Laboratório de Farmacologia localizado no Centro Universitário UniFacisa localizado na cidade de Campina Grande.

Para tanto, foram adquiridas 10 amostras em diferentes pontos das cidades supracitadas, sendo eles, 5 na cidade de Campina Grande e as outras 5 na cidade de Pocinhos. Todas as amostras foram mantidas em suas embalagens originais utilizadas para comercialização. Para identificação das análises, as amostras foram identificadas pelas respectivas siglas: Feira Livre (FL1-PC/ FL2-PC/ FL3-CG/ FL4-CG), loja de produtos naturais (LPN1-PC/ LPN2-PC/ LPN3-CG/ LPN4-CG) e supermercados (SP1-PC e SP2-CG).

\subsection{Realização dos testes}

\subsubsection{Teste de ativação do amido}

O primeiro teste empregado foi o teste de ativação do amido, no qual foram utilizados para cada análise um becker contendo $8 \mathrm{gr}$ da cúrcuma em pó para $200 \mathrm{ml}$ de água, em seguida a mistura foi homogeneizada e aquecida até levantar fervura, logo após, desligou-se a chapa aquecedora e por fim, observou se a cúrcuma estava bem dissolvida e líquida ou se apresentava como um líquido encorpado devido a presença do amido. Para as outras nove amostras repetiu o mesmo processo.

\subsubsection{Teste de reação do lugol}

Esse teste é uma reação colorimétrica qualitativa, realizada conforme metodologia do Instituto Adolfo Lutz (2008). Indica a presença de amido, e considera-se positiva quando a coloração final da solução for violeta ou azul.

Da solução realizada para o teste anterior, contendo $8 \mathrm{gr}$ da cúrcuma em $200 \mathrm{ml}$ de água, foram $2 \mathrm{ml}$ e colocados em tubos de ensaio e neles foram adicionadas 2 gotas da solução de lugol e em seguida, homogeneizados, e analisadas as mudanças de coloração das amostras. O mesmo ensaio foi realizado com uma solução pura com amido diluído em água para comparação.

\section{Resultados e Discussão}

Das 5 amostras adquiridas na cidade de Pocinhos - PB duas foram obtidas na Feira Livre (Figura 1-A* I-FL1 e 1-B* II-FL2), duas obtidas em Lojas de Produtos Naturais (Figura 1-C* V-LPN1 e 1-D* X-LPN4) e uma foi adquirida em Supermercado (Figura 1-E* VI-SP1).

Figura 1 - Amostras em suas embalagens originais de comercialização adquiridas na cidade de Pocinhos-PB. 


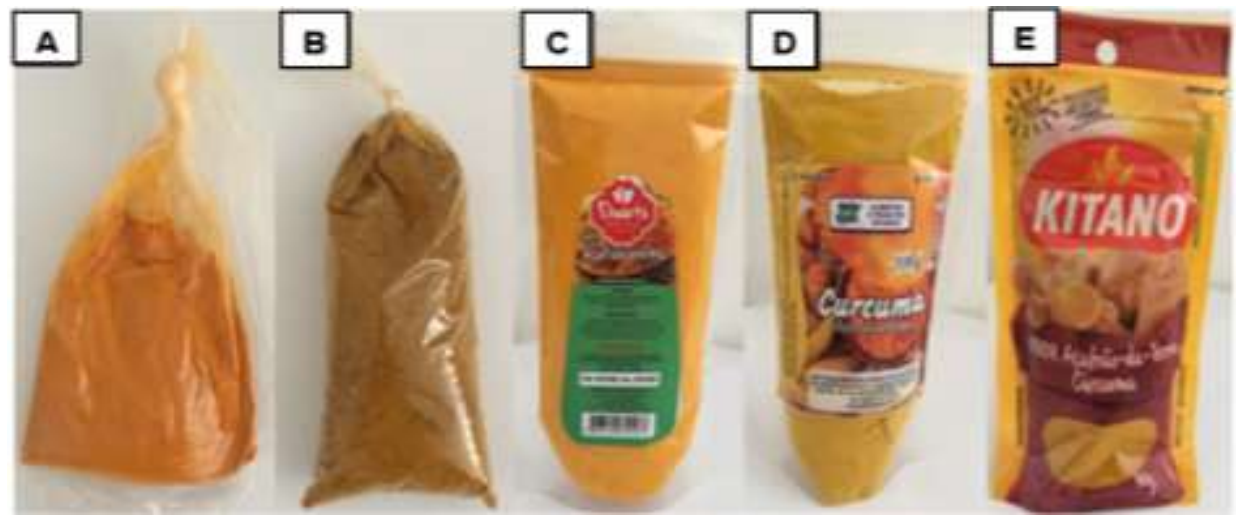

Fonte: Autores (2021).

Na cidade de Campina Grande-PB foram adquiridas outras 5 amostras. Duas delas foram obtidas na Feira Livre (Figura 2-F* III-FL3 e 2-G* IV-FL4), outras duas obtidas em Lojas de Produtos Naturais (Figura 2-H* VIII-LPN2 e 2-I* IX LPN3) e a uma em Supermercado (Figura 2-J* VII-SP2).

Figura 2 - Amostras em suas embalagens originais de comercialização adquiridas na cidade de Campina Grande-PB.

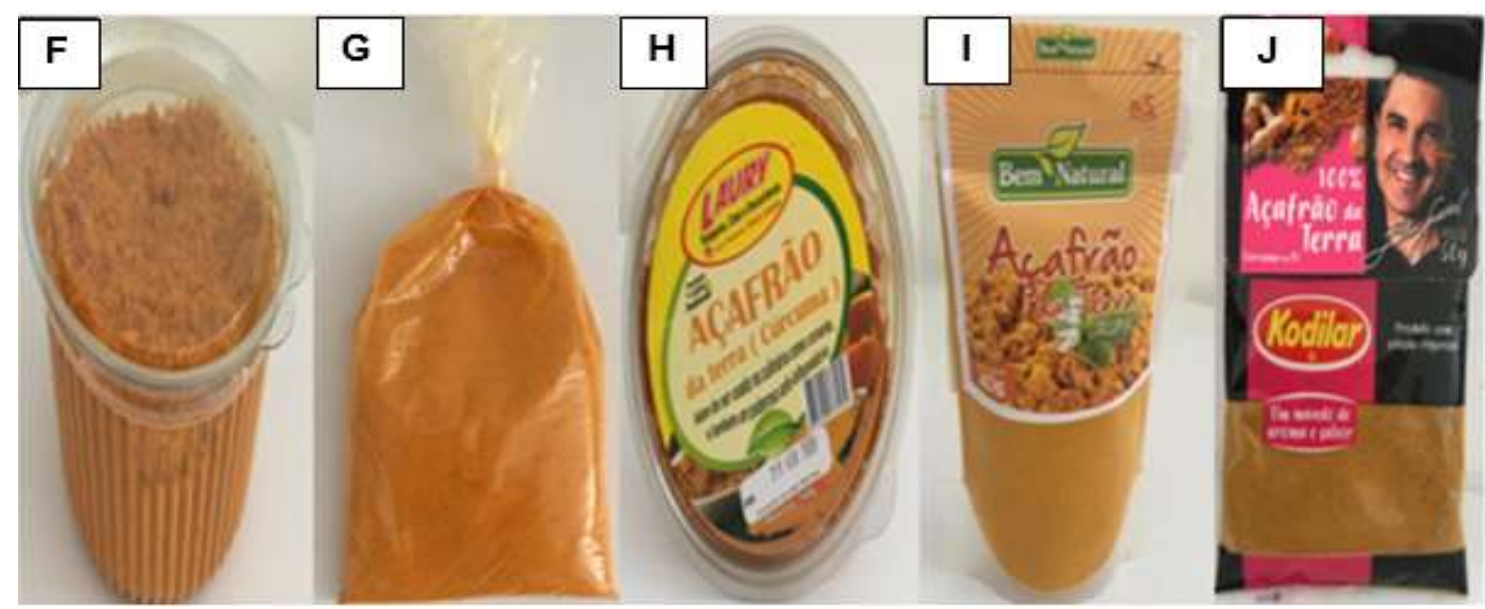

Fonte: Autores (2021).

No teste de ativação do amido, as amostras foram aquecidas, para assim, ativarem o amido, a presença do mesmo pode ser constatada se a solução apresentar aspecto encorpado.

Após realização do teste de ativação do amido nas amostras adquiridas na cidade de Pocinhos-PB, observou-se que todas as amostras ficaram com um aspecto líquido e bem dissolvido, denotando assim, com esse teste ausência de amido nas amostras, conforme (Tabela 1). 
Research, Society and Development, v. 10, n. 7, e11010716233, 2021

(CC BY 4.0) | ISSN 2525-3409 | DOI: http://dx.doi.org/10.33448/rsd-v10i7.16233

Tabela 1 - Resultados do teste de ativação do amido de amostras de cúrcuma em pó da cidade de Pocinhos - PB.

\section{AMOSTRAS POCINHOS - PB}

\begin{tabular}{cccccc}
\hline & FL1 & FL2 & LPN 1 & SP1 & LPN 4 \\
\hline $\begin{array}{c}\text { TESTE DE } \\
\text { ATIVAÇAO DO } \\
\text { AMIDO }\end{array}$ & NEGATIVO & NEGATIVO & NEGATIVO & NEGATIVO & NEGATIVO \\
\hline
\end{tabular}

Fonte: Autores (2021).

Ao analisar as amostras oriundas da cidade de Campina Grande-PB, verificou-se que a amostra FL4-CG foi à única amostra reprovada no teste de ativação de amido (Tabela 2).

Tabela 2 - Resultados do teste de ativação do amido de amostras de cúrcuma em pó da cidade de Campina Grande- PB.

AMOSTRAS CAMPINA GRANDE - PB

\begin{tabular}{cccccc}
\hline & FL3 & FL4 & SP2 & LPN 2 & LPN 3 \\
$\begin{array}{c}\text { TESTE DE } \\
\text { ATIVAÇAO DO } \\
\text { AMIDO }\end{array}$ & NEGATIVO & POSITIVO & NEGATIVO & NEGATIVO & NEGATIVO \\
\hline
\end{tabular}

Fonte: Autores (2021).

Essa amostra ao final do teste apresentou-se como um líquido bem espesso e aspecto encorpado, com dificuldade de se desprender das paredes do becker e da espátula. As demais amostras durante todo o ensaio apresentaram-se como líquido fino, sem aderir nas paredes do becker ou na espátula, exibindo aspecto bem fluido, conforme (Figura 3).

Figura 3 - Amostras após o teste de ativação do amido.

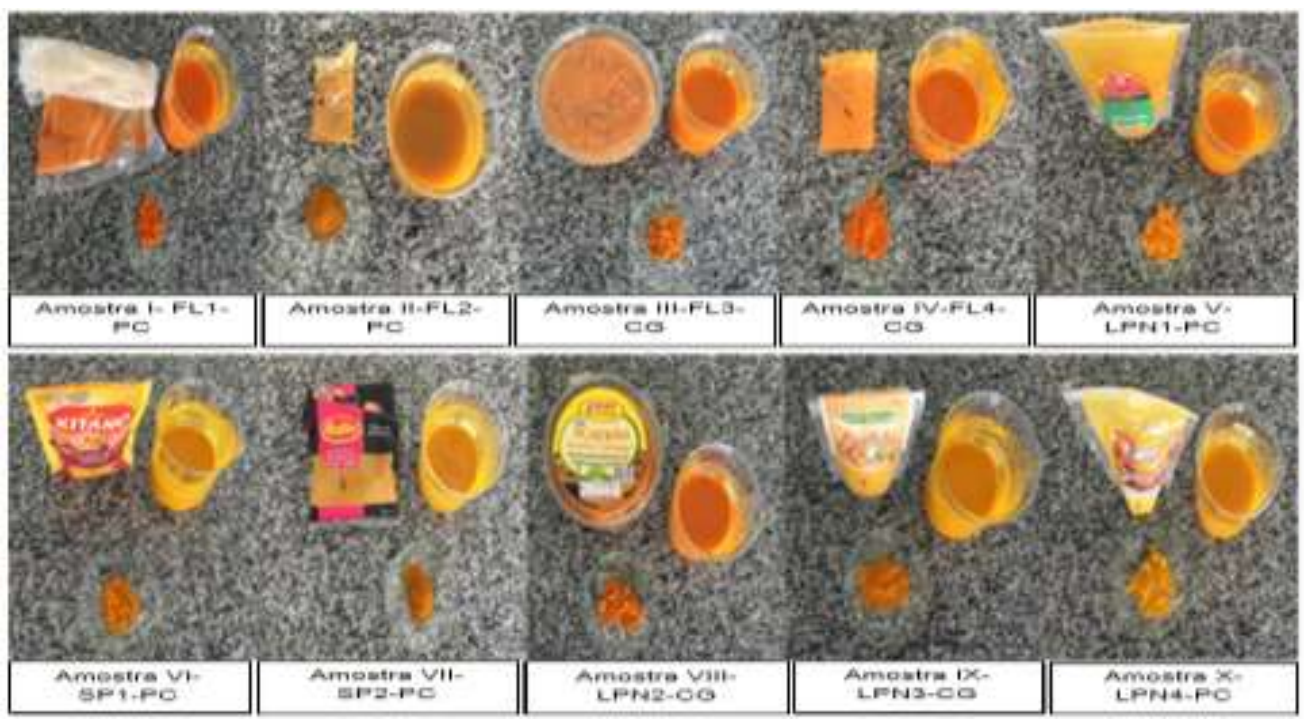

Fonte: Autores (2021). 
Já o teste do lugol objetiva identificar através de uma reação colorimétrica a presença do amido, que em caso positivo observa-se uma mudança de coloração na solução de cor violeta ou azul.

Após realização do teste colorimétrico nas amostras adquiridas nas cidades de Pocinhos-PB e Campina GrandePB, observou-se que as amostras FL1-PC / V- LPN 1-PC/ FL3-CG e FL4-CG apresentaram mudança de coloração, como mostra (Figura 4). Logo a amostra FL4-CG, foi à única reprovada nos dois testes.

Figura 4 - Amostras do pó da cúrcuma após o teste de reação do lugol, observando mudança na coloração.

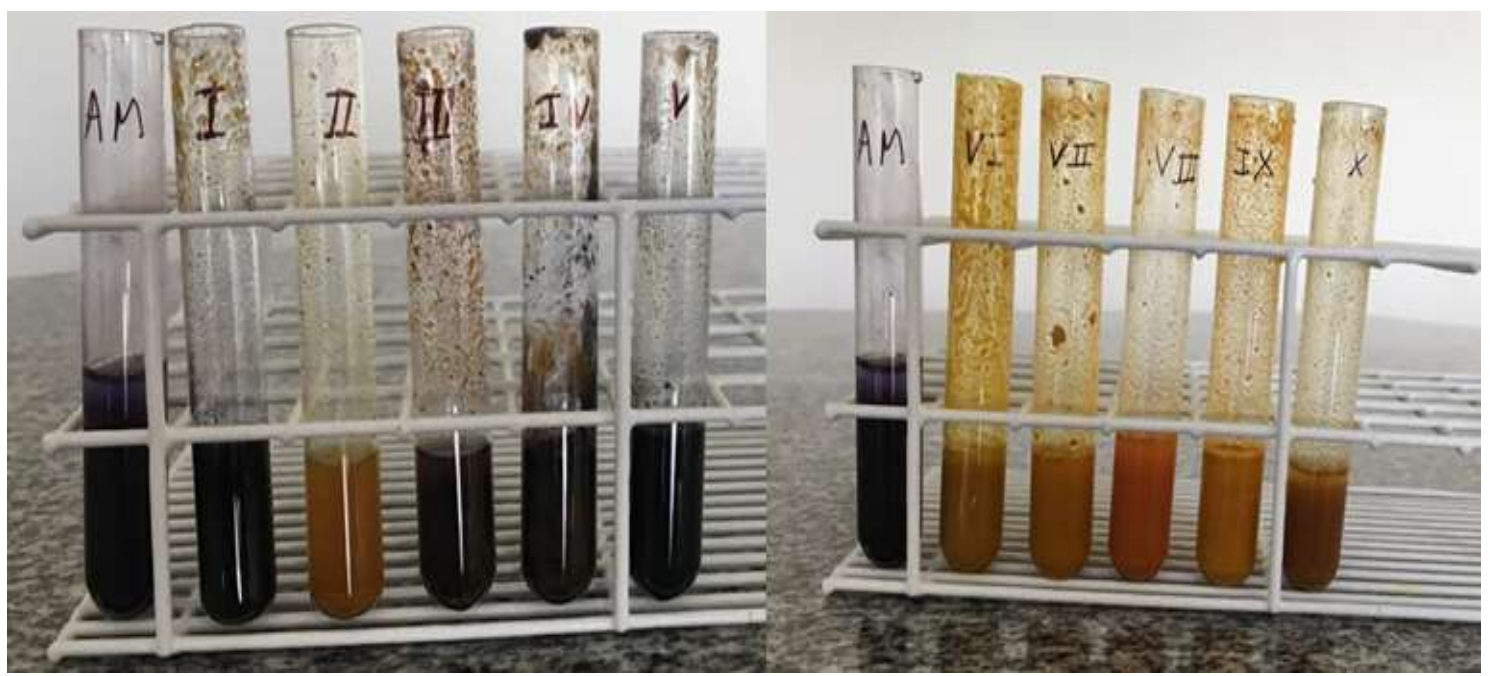

Fonte: Autores (2021).

A amostra FL 2- PC foi a única adquirida em feira livre que não foi reprovada em nenhum dos testes para identificação de adulteração por adição de amido, no entanto, seu pó apresentava-se com uma cor castanha clara e cheiro característico do condimento cominho (Cuminum cyminum L.), o que é uma prática bastante comum a troca parcial ou total de uma especiaria com características parecidas por outra de menor valor. Em trabalhos de Pauli-Yamada, et al. 2020 e Sousa 2017, foram retratados algumas especiarias que não faziam parte da composição original, sendo adicionadas ao produto comercializado.

De acordo com Silvis, et al. (2017), a cúrcuma em pó é um grande alvo para possíveis adulterações devido a diversos fatores: um deles é a falta de análises para detectar as adulterações. A mesma autora ressalta que essas especiarias em pó são as mais sujeitas a ações fraudulentas, pois na sua produção as etapas de moagem ou trituração do seu processo transformam tanto o produto quanto o adulterante, em pó, por essa razão, a longa e complexa cadeia de distribuição, na qual possibilita que a adulteração seja feita em diversas etapas, torna sua identificação uma tarefa ainda mais complicada.

As especiarias, tais como, cúrcuma (açafrão das índias); o açafrão; páprica; pimenta etc., fazem parte uma classe atrativa para os fraudadores, porque têm um valor comercial elevado (Silvis, et al. 2017). Por ser considerada uma das especiarias mais cara do mundo, o açafrão, vem sendo referido em estudos recentes como a especiaria mais adulterada (Johnson, 2014; Cérou, 2018).

Diante disso, é conhecida por fazer parte do grupo de alimentos vulneráveis à adulteração, podendo ser ocasionada pela adição de matérias vegetais estranhos ao produto, com a intenção de aumentar o seu peso/volume aparente, tais como: ingredientes vegetais baratos (por exemplo, sementes de papaia); amidos; gesso; calcário; entre outros (Cérou, 2018; Food Safety Helpline, 2018). 
Procedimentos como esses podem resultar em um produto de baixa qualidade, com menor ação benéfica à saúde ou até mesmo ser nocivo ao consumidor (Bansal, et al. 2017). Em razão da cúrcuma exibir diversos benefícios à saúde, sua utilização para fins medicinais vem aumentando de maneira elevada, em contrapartida, quando a mesma sofre algum procedimento adulterante, consequentemente a qualidade do produto diminui, da mesma maneira que as propriedades medicinais, o que acaba lesando o consumidor.

Graças aos múltiplos benefícios é amplamente utilizado na indústria alimentícia em forma de tempero, corante, suplemento dietético, inibidor de apetite, termogênico no controle da obesidade entre outras, usado na aromaterapia, na medicina tradicional como medicamento caseiro na forma de chá, decocção, maceração e infusão, na fitoterapia utilizando-se as partes secas e frescas para extratos, chás, óleos, pomadas, comprimidos e cremes, na indústria de cosméticos, constituído por óleos essenciais, cremes e pomadas para a pele, bem como, na indústria farmacológica como antioxidante, anticoagulante, anti-inflamatório, antitumoral, antiviral, anticancerígeno, neuroprotetor e outras fisiopatologias (Ferreira, 2014; Sahoo, et al. 2016, Moretes \& Geron, 2019).

Em decorrência disso, a má qualidade e a presença de adulterações acaba interferindo na eficácia e segurança desses produtos, reforçando cada vez mais a importância da implementação de um serviço de vigilância ainda mais eficiente (BraghinI, et al. 2015).

Estudo desenvolvido por Brito 2020, foram utilizados imagens hiperespectrais para determinar a quantidade de amido presente em amostras de cúrcuma, nas quais, apresentaram uma relação entre a adição de amido nas amostras em estudo, corroborando com o presente estudo e evidenciando a recorrência desse tipo de adulteração.

Em análises realizadas por Pauli-Yamad, et al. (2020), observou-se que na especiaria da páprica em pó, o amido de milho foi o ingrediente adicionado com maior frequência (85\%) entre os adulterantes. Tal relevância baseia-se no fato de que quando adicionados esses componentes de aparência similar ao produto original eles são de difícil distinção e detecção, tornando um desafio continuo das autoridades encarregadas (Moore; Spink; Lipp, 2012; Johnson, 2014; Sousa, 2017).

Nesse mesmo contexto, em pesquisa realizada por Sousa (2017), para autenticação de pimentas (Piper nigrum L.), as amostras adulteradas representavam 47,8\%, e dessa porcentagem $24,1 \%$ foram adulteradas com farinha de milho, evidenciando a utilização dessa substância como adulterante em diversas especiarias.

Essa facilidade de adulteração se torna ainda maior em produtos vendidos de maneira informal, como é o caso da cúrcuma (Silva Neto; Leite, Aguiar, 2020). Isso porque, os produtos manufaturados que são comercializados em menor proporção e de forma caseira, como em feiras públicas locais, na maioria das vezes, apresentam apenas uma embalagem de acondicionamento, sem quaisquer informações de sua composição ao consumidor (Almeida, et al. 2017).

A falta dessas informações na embalagem dificulta uma aquisição segura dos produtos, a exemplo do pó da cúrcuma vendido em feiras livres, como se observa nas amostras FL1-PC/ FL 2- PC/ FL 3-CG e FL 4-CG (Figura 5). Com a ocultação dessas informações acerca da composição dos alimentos os consumidores não conseguem ter consciência e mensurar o que estão adquirindo. 
Figura 5 - Amostras obtidas em Feira Livre das cidades de Campina Grande-PB e Pocinhos-PB, FL1-PC/ FL2- PC/ FL3-CG/ FL4-CG, respectivamente.

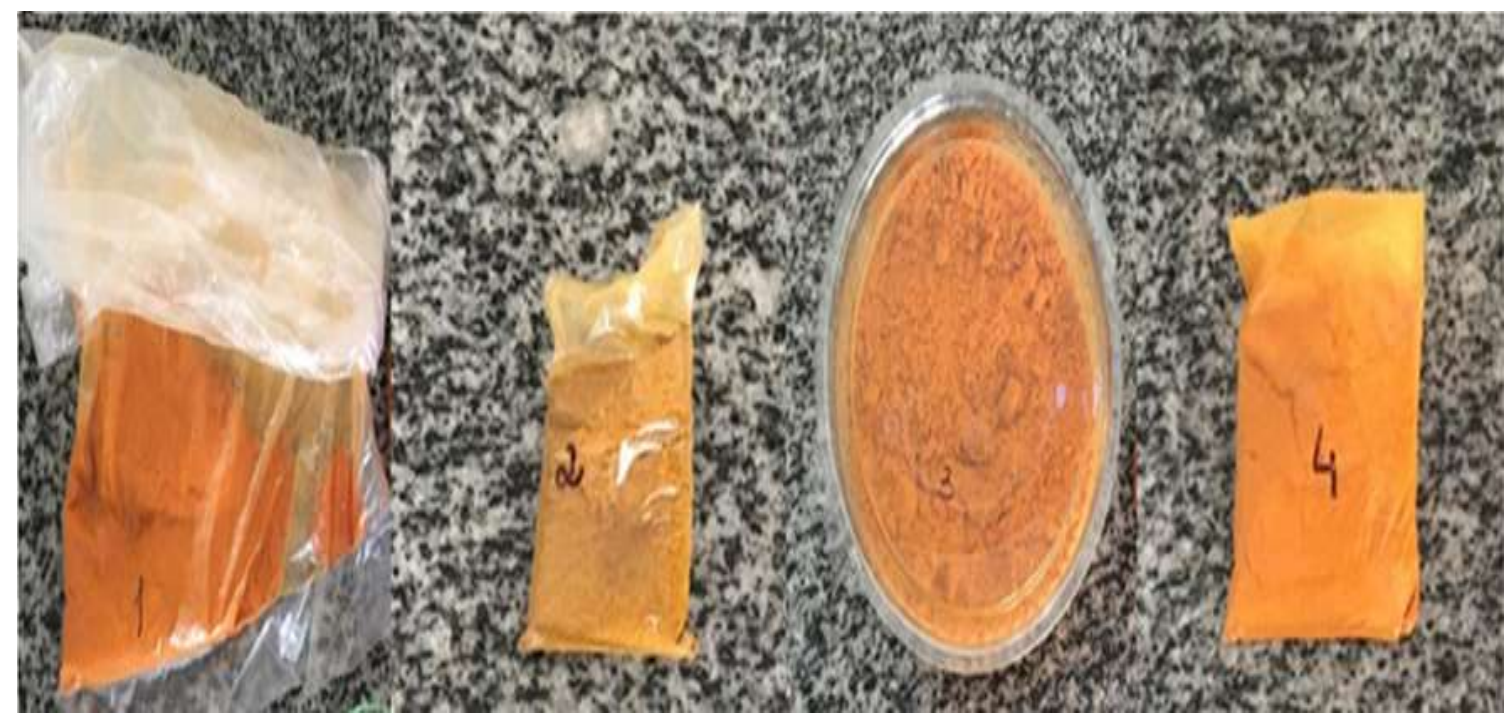

Fonte: Autores (2021).

Quando um produto se apresenta sem nenhum tipo de marcação e a granel, o mesmo possui maior possibilidade de sofrer adulterações, diferente de um produto que se apresente na forma pré-embalada, onde esse risco diminui consideravelmente (IFS, 2018). Isso se explica pelo fato de quanto maior for à facilidade de se adulterar/falsificar e em combinação tiver uma menor probabilidade de detecção, consequentemente maior será a oportunidade do fraudador em realizar suas práticas ilícitas (Van Ruth; Huisman; Luning, 2017).

Segundo Rodrigues, et al. (2020), em análise microscópica da cúrcuma, foi detectado a presença Zea mays (milho) em $30,3 \%$ das amostras, bem como, as listas de ingredientes das amostras analisadas continham apenas a especiaria referente ao produto, contrastando com a pesquisa de elementos histológicos, que verificou a presença de componentes da Zea mays (milho), advertindo para a possibilidade de adulteração do produto.

De acordo com as Normas Técnicas Especiais para alimentos e bebidas (Brasil, 1978) são considerados como condimento vegetal, aqueles constituídos de especiarias originais e puras, bem como, devendo estar limpas e sãs, correspondendo às suas características botânicas normais e não possuir elementos vegetais estranhos a espécie de partes da planta de origem, que não caracterize como condimento vegetal. Diferente do condimento preparado, que também é constituído de especiarias originais e outros ingredientes, que necessitam estar em perfeita conservação, podendo ser permitida a adição de amido na quantidade máxima de $10 \%$.

Diante da diferenciação acima a legislação sanitária de rotulagem de alimentos, RDC ANVISA nº 259/2002, prevê a obrigatoriedade da declaração de lista de ingredientes no rótulo dos alimentos embalados quando o produto é composto por mais de um ingrediente (Brasil, 2002).

Tal norma é utilizada para verificação da conformidade dos alimentos em relação à sua identidade, portanto, nesse estudo, as amostras FL1-PC/ FL2- PC/ FL3-CG/ FL4-CG e LPN1- PC, estavam em desconformidades por não estarem condizentes com a denominação de venda "cúrcuma" ou por apresentarem componentes não declarados na lista de ingredientes.

Das amostras citadas a cima a LPN 1-PC era a única que possuía rótulo e nele constava a indicação de "Produto $100 \%$ puro" (Figura 6), no entanto, essa amostra foi reprovada no teste do lugol comprovando assim a existência de outros compostos na amostra. 
Figura 6 - Rótulo da amostra V-LPN 1-PC.

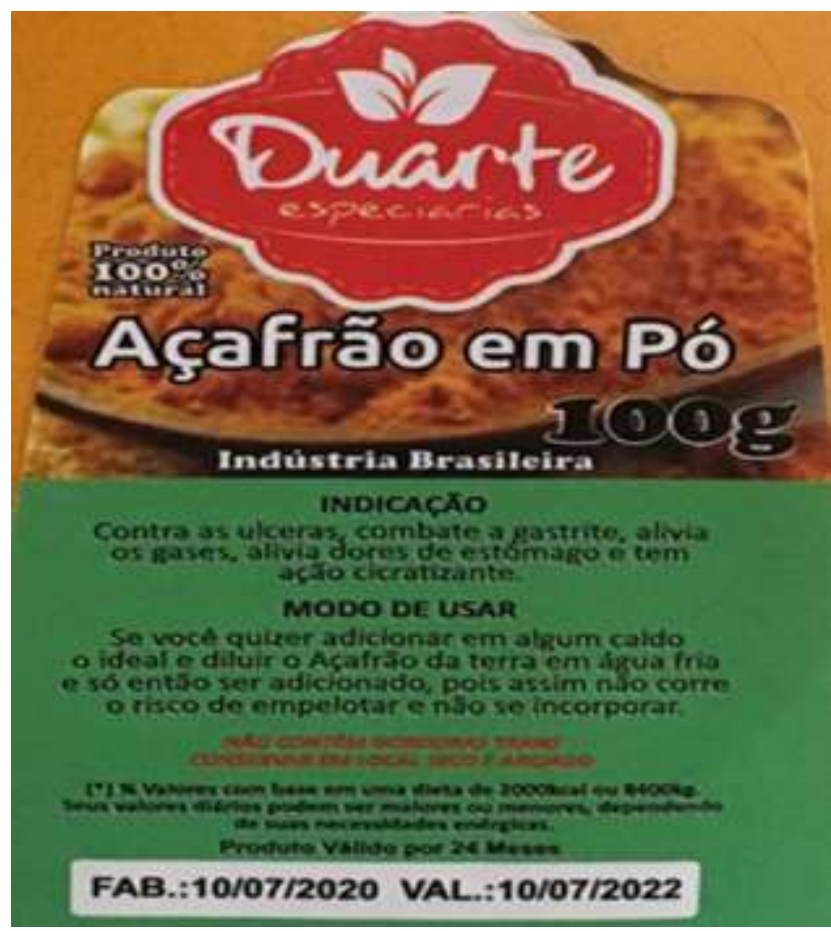

Fonte: Autores (2021).

Portanto, é importante garantir a efetividade e a qualidade desses produtos alimentícios, para a proteção do consumidor (Van Asselt; Banach; Van Der Fels-Klerx, 2018), pois muitos casos de adulteração com ervas e especiarias têm sido relatados recentemente (Galvin-King; Haughey; Elliott, 2018).

As demais amostras FL1-PC, FL2-PC, FL3-CG e FL4-CG, não apresentaram qualquer tipo de informação a respeito da composição do produto, contrariando a RDC ANVISA n 259/2002, impossibilitando a identificação pelo consumidor de informações básicas que são essenciais para seu consumo e que tem por finalidade garantir a sua segurança alimentar (Brasil, 2002).

Sendo um direito do consumidor dispor de informações legítima acerca dos alimentos e ingredientes alimentares, tal como estipulado no decreto $\mathrm{n}^{\mathbf{0}} 4.680 / 2003$. Tendo como principais objetivos precaver práticas enganadoras e fraudulentas, adulteração e outras práticas que possam influenciar o consumidor a confusão ou erro acerca do mesmo (Brasil, 2003; CuberoLeon; Peñalver; Maquet, 2014).

Dessa maneira, os rótulos alimentícios proporcionam garantias aos consumidores, como também, promove uma melhoria na estética da embalagem. Essa identificação presente nos alimentos é de extrema importância, uma vez que, facilita para os órgãos fiscalizadores competentes, na hora de analisar em loco os alimentos pelo seu rótulo (Silva Neto; Leite, Aguiar, 2020). Se todos os alimentos contivessem rótulos os consumidores ficariam mais esclarecidos por meio das informações contidas nos mesmos (De Morais, et al. 2018).

De acordo com Souza, et al. (2017) as informações acerca dos alimentos se torna de extrema necessidade, visto que sua ausência acarretara uma maior dificuldade para as intervenções de políticas públicas e investigações científicas para que medidas possam ser adotadas.

As técnicas para fraudar especiarias estão muito avançadas, mas no geral, a forma mais precisa para demonstrar que a especiaria foi fraudada é realizar análises químicas, bioquímicas e microscópicas que comprovam a presença de um corpo estranho. 
Dhanya e Sasikumar, (2010) apresentam alguns exemplos de análises de deteç̧ão, tais como, cromatografia líquida de alta performnce, cromatografia em camada fina, cromatografia gasosa, espectroscopia líquida e gasosa, análises microscópicas, eletroforese capilar, entre outras.

No entanto, segundo estes mesmos autores, embora essas técnicas sejam eficazes, esses métodos não são convenientes para análises de rotina de uma indústria de alimentos, pois tem alto custo e são demorados.

Por isso, torna-se fundamental a aplicação de um controle extremamente rigoroso pelas autoridades responsáveis, para controlar as práticas de adulteração e/ou fraude alimentar, nas quais possuem grandes impactos tanto a nível econômico quanto na defesa do consumidor (Sol, et al. 2015).

\section{Conclusão}

A partir da análise dos dados, observou-se que das 4 amostras comercializadas em feira livre 3 estavam adulteradas com amido e das 4 comercializadas em lojas de produtos naturais uma apresentou o mesmo tipo de adulteração. As duas amostras vendidas em supermercados não apresentaram nenhuma adulteração.

Diante do alto índice de adulteração encontrado nas amostras desse estudo, acende-se um alerta sobre a necessidade de implementação de mecanismos de controle para tais produtos que estão sendo vendidos deliberadamente, em especial porque a cúrcuma vem sendo consumida para fins medicinais e tal adulteração ocasiona a diminuição das suas propriedades terapêuticas.

Ademais, se faz necessário outras metodologias que quantifiquem os constituintes adulterantes, como as análises físico-químicas para determinação de cinzas (resíduo mineral fixo), cinzas insolúveis em ácido clorídrico a 10\% e extrato alcoólico, bem como, outros empregados, para que os resultados possam ser comparados com os valores estabelecidos pela legislação.

Por fim, destaca-se como principal limitação do estudo a escassez de pesquisas anteriores que abordem sobre a adulteração no pó da cúrcuma pela adição de amido, bem como, em outras especiarias. Assim, espera-se com essa produção contribuir para a construção acerca de estudos futuros relacionados à adulteração na Cúrcuma longa.

\section{Referências}

Almeida, J. M., Alves, T. C. O., Carmo, A. S., \& Martins, P. C. (2017). Avaliação da rotulagem em alimentos caseiros comercializados sem rótulos em mercado público de João Pessoa-PB. Revista Brasileira de Agrotecnologia, 7(2), 01-07.

Alonso, J. (2016). Tratado de Fitofármacos e Nutracêuticos [Tradução Luciano Prado da Silva, Maria Edith Barbagelata et al]. São Paulo: Ac Farmacêutica.

Bansal, S., Singh, A., Mangal, M., Mangal, A. K., \& Kumar, S. (2017). Food adulteration: Sources, health risks, and detection methods. Critical reviews in food science and nutrition, 57(6), 1174-1189.

Barreto, A., Cruz-Tirado, J. P., Siche, R., \& Quevedo, R. (2018). Determination of starch content in adulterated fresh cheese using hyperspectral imaging. Food Bioscience, 21, 14-19.

Braghini, F., Souza, F., Gonçalves, R. A., Oliveira, A. J., \& Gonçalves, J. E. (2015). Avaliação da qualidade de plantas medicinais comercializadas na cidade de Maringá-PR. Enciclopédia Biosfera, 11(21).

Brasil (1978). Comissão Nacional de Normas e Padrões para Alimentos. Resolução - CNNPA n ${ }^{\circ} 12$, de 24 de julho de 1978. Aprova as normas técnicas especiais. Diário Oficial [da] República Federativa do Brasil, Brasília. http://portal.anvisa.gov.br/wps/wcm/connect/e57b7380474588a39266d 63fbc4c6735/RESOLUCAO_12_1978.pdf?MOD=AJPERE

Brasil (2003). Decreto $n^{\circ} 4.680$, de 24 de Abril de 2003. Regulamenta o direito à informação, assegurado pela Lei ${ }^{\circ} 8.078$,de 11 de Setembro de 1990 , quanto aos alimentos e ingredientes alimentares destinados ao consumo humano ou animal que contenham ou sejam produzidos a partir de organismos geneticamente modificados, sem prejuízo do cumprimento das demais normas aplicáveis. Diário Oficial da União República Federativa do Brasil, Brasília.

Retirado de http://www.planalto.gov.br/ccivil_03/decreto/2003/d4680.htm

Brasil (2002). Ministério da Saúde. Agência Nacional de Vigilância Sanitária. Aprova o Regulamento técnico sobre rotulagem de alimentos embalados. Resolução $\mathrm{n}^{\circ}$ 259, 20 de setembro de 2002. Aprova o regulamento 38 técnico para rotulagem de alimentos embalados. Diário Oficial da União, Poder Executivo, Brasília. http://www.anvisa.gov.br 
Brasil (2009). Ministério da Saúde. Relação Nacional de Plantas Medicinais de Interesse do SUS - RENISUS. http://portalarquivos2.saude.gov.br/images/pdf/2014/maio/07/renisus.pdf

Brito, R. J. D. (2020). Utilização de imagens hiperespectrais para controle de qualidade de amostras de Curcuma Longa.

Calixto, J. B. (2019). O papel dos produtos naturais na descoberta de medicamentos modernos. Anais da Academia Brasileira de Ciências, 91, 1-7.

Cérou, M (2018). 51\% de fraudes détectées dans les épices. Process Alimentaire. http://www.processalimentaire.com/Qualite/51-de-fraudes-detecteesdansles-epices-34361

Cubero-Leon, E., Peñalver, R., \& Maquet, A (2014). Review on metabolomics for food authentication. Food Research International, $60,95-107$.

De Moraes, André Luiz Santos. et al (2018). Teor de sódio em alimentos consumidos pela população do estado da Paraíba. Revista de Ciências da Saúde 16, 51-57. https://revista.facene.com.br/index.php/revistane/article/view/20/32

Dhanya, K., \& Sasikumar, B. (2010). Molecular marker based adulteration detection in traded food and agricultural commodities of plant origin with special reference to spices. Current Trends in Biotechnology \& Pharmacy, 4(1).

Ferreira, Priscylla Paulina (2014). Extração, caracterização e aplicação de fécula de açafrão (Curcuma longa L.) no desenvolvimento de biscoito. 2014, 51 f. Dissertação (Mestrado em Ciência e Tecnologia de Alimentos), Escola de Agronomia da Universidade Federal de Goiás, Goiânia, Goiás, Brasil

Food safety helpline (2018). FSSAI Issues Guidance Note on Safe Ground Spices. https://foodsafetyhelpline.com/safe-ground-spices/

Galvin-King, P., Haughey, S. A., Elliott, C. T (2018). Fraude de ervas e especiarias, os drivers, desafios e detecção. Food Control, 88, 85-97.

Gil, A. Como elaborar projetos de pesquisa. (5a ed.), Atlas.

Global food safety initiative (GFSI) (2018). Tackling Food Fraud Through Food Safety Management System. https://mygfsi.com/wpcontent/uploads/2019/09/Food-Fraud-GFSI-Technical-Document.pdf

GMA Science \& Education Foundation (GMA-SEF) (20018). Food and Consumer Product Fraud: Prevention and Mitigation https://consumerbrandsassociation.org/newsblog/?fwp_news_types=blog\&fwp_posts_per_page=undefined >

Instituto Adolfo Lutz - IAL (2008). Normas analíticas do Instituto Adolfo Lutz: métodos químicos e físicos para análise de alimentos. (5a ed.) p.1020.

International Featured Standards (IFS) (2018). Development. Standards Product Fraud. https://www.ifscertification.com/index.php/en/blog/ifs-developmentblog

Jonhson, Renée (2014). Food Fraud and economically motivated adulteration of food and food ingredients. Congressional Research Service, Washington. https://fas.org/sgp/crs/misc/R43358.pdf

Lucas, Ricardo Rodrigues. et al (2016). Fitoterápicos aplicados à obesidade. DEMETRA: Alimentação, Nutrição \& Saúde, 11, $473-492$.

Moore, Jeffrey C., SPINK, John, LIPP, Markus (2012). Development and application of a database of food ingredient fraud and economically motivated adulteration from 1980 to 2010. Journal of Food Science, 77, 118-126. https://onlinelibrary.wiley.com/doi/epdf/10.1111/j.1750-3841.2012.02657.x

Moretes, D. N., Geron, V. L. M. G. (2019). Os benefícios medicinais da Curcuma longa L. (açafrão da terra). Revista Científica da Faculdade de Educação e Meio Ambiente, 10, 106-114. http://www.faema.edu.br/revistas/index.php/Revista-FAEMA/article/view/767/767

Muniz, R. M., Carvalho Caminha et al (2013) Plantas Medicinais Da Renisus De Atuação Central. Infarma-Ciências Farmacêuticas, 24, 75-80. http://www.revistas.cff.org.br/?journal=infarma\&page=article\&op=view\&path $\% 5 \mathrm{~B} \% 5 \mathrm{D}=361 \&$ path $\% 5 \mathrm{~B} \% 5 \mathrm{D}=350$

Pauli-Yamada, L. F. et al (2020). Estudo microscópico de páprica (Capsicum annuum L.): detecção de fraudes e matérias estranhas. Vigilância Sanitária em Debate: Sociedade, Ciência \& Tecnologia. São Paulo, 8, 1-6.

Pedrete, T. de A. et al (2018). Caracterização proteômica e avaliação de toxicidade de plantas hipoglicemiantes: estudo dos extratos protéicos de Bauhínias e Chrysobalanus icaco 141 f. Tese (Doutorado em Saúde Pública e Meio Ambiente) - Escola Nacional de Saúde Pública Sergio Arouca, Fundação Oswaldo Cruz, Rio de Janeiro.

Rodrigues, M. L. et al (2020). Pesquisa de matérias estranhas e adulterações em cúrcuma e noz-moscada comercializadas no estado de São Paulo. $7^{\circ}$ Simpósio de Segurança Alimentar. http://schenautomacao.com.br/ssa7/envio/files/trabalho3_328.pdf

Sahoo, A. et al (2016). Resequencing of Curcuma longa L. cv. Kedaram through transcriptome profiling reveals various novel transcripts. Genomics data, 9 $160-161$.

Silva Neto, I. F., et al (2020). Análise bromatológica do teor sódio no condimento em pó açafrão (Curcuma longa L.). Alimentos: Ciência, Tecnologia e Meio Ambiente, 1, 173-183.

Silvis, I. C. J. et al (2017). Assessment of food fraud vulnerability in the spices chain: An explorative study. Food Control, 81, 80-87.

Sol, M. et al (2015). Deteção de fraudes alimentares em carne - análise de ADN. Riscos e Alimentos, 9, 8-10

SOUSA, Ana Isabel Ferreira da Costa. Desenvolvimento de marcadores de ADN para autenticação de pimentas (Piper nigrum L.). 2017. Dissertação (Mestrado em Controlo de Qualidade na Especialidade Água e Alimentos), Universidade do Porto, Porto, Portugal.

Souza, Amanda de Moura. et al (2017). Redução do teor de sódio em alimentos processados: estamos no caminho certo? Cad. Saúde Pública, 33 , eCA010117. 
Research, Society and Development, v. 10, n. 7, e11010716233, 2021

(CC BY 4.0) | ISSN 2525-3409 | DOI: http://dx.doi.org/10.33448/rsd-v10i7.16233

Spink, John, Moyer, Douglas C (2011). Defining the public health threat of food fraud. Journal of food science, 76, R157-R163. https://onlinelibrary.wiley.com/doi/epdf/10.1111/j.1750-3841.2011.02417.x

Van Asselt, E. D., Banach, J. L., Van Der Fels-Klerx, H. J (2018). Prioritization of chemical hazards in spices and herbs for European monitoring programs. Food Control, 83, 7-17.

Van Ruth, S. M., Huisman, W., \& Luning, P. A (2017). “Food fraud vulnerability and its key factors”. Trends in Food Science \& Technology, 67, 70-75. 